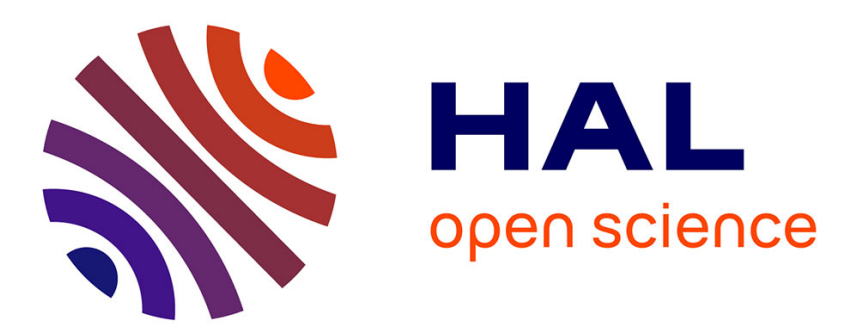

\title{
Continuous velocity fields for collapse and blow-out of a pressurized tunnel face in purely cohesive soil
}

Guilhem Mollon, Daniel Dias, Abdul-Hamid Soubra

\section{To cite this version:}

Guilhem Mollon, Daniel Dias, Abdul-Hamid Soubra. Continuous velocity fields for collapse and blowout of a pressurized tunnel face in purely cohesive soil. International Journal for Numerical and Analytical Methods in Geomechanics, 2013, 37 (13), pp.2061-2083. 10.1002/nag.2121 . hal-01005994

\section{HAL Id: hal-01005994 \\ https://hal.science/hal-01005994}

Submitted on 29 Oct 2018

HAL is a multi-disciplinary open access archive for the deposit and dissemination of scientific research documents, whether they are published or not. The documents may come from teaching and research institutions in France or abroad, or from public or private research centers.
L'archive ouverte pluridisciplinaire HAL, est destinée au dépôt et à la diffusion de documents scientifiques de niveau recherche, publiés ou non, émanant des établissements d'enseignement et de recherche français ou étrangers, des laboratoires publics ou privés. 


\title{
Continuous velocity fields for collapse and blowout of a pressurized tunnel face in purely cohesive soil
}

\author{
Guilhem Mollon $^{1, *}{ }^{\dagger}$, Daniel Dias ${ }^{2}$ and Abdul-Hamid Soubra ${ }^{3}$ \\ ${ }^{1} 3 S R$, Grenoble-INP, UJF-Grenoble 1, CNRS UMR 5521, Grenoble, France \\ ${ }^{2}$ LGCIE Equipe Géotechnique, Bât. J.C.A. Coulomb, Domaine scientifique de la Doua, INSA Lyon, Villeurbanne cedex, France \\ ${ }^{3}$ University of Nantes, Civil Engineering Department, Saint-Nazaire cedex, France
}

Face stability analysis of tunnels excavated under pressurized shields is a major issue in real tunnelling projects. Most of the failure mechanisms used for the stability analysis of tunnels in purely cohesive soils were derived from rigid block failure mechanisms that were developed for frictional soils, by imposing a null friction angle. For a purely cohesive soil, this kind of mechanism is quite far from the actual velocity field. This paper aims at proposing two new continuous velocity fields for both collapse and blowout of an air-pressurized tunnel face. These velocity fields are much more consistent with the actual failures observed in undrained clays. They are based on the normality condition, which states that any plastic deformation in a purely cohesive soil develops without any volume change. The numerical results have shown that the proposed velocity fields significantly improve the best existing bounds for collapse pressures and that their results compare reasonably well with the collapse and blowout pressures provided by a commercial finite difference software, for a much smaller computational cost. A design chart is provided for practical use in geotechnical engineering.

KEY WORDS: limit analysis; tunnel face stability; purely cohesive soils; continuous velocity field; face collapse

\section{INTRODUCTION}

In real tunnelling projects using a pressurized shield, the main issues of the designer are the face instabilities and the possible ground settlements that the excavation may induce. This paper only focuses on tunnel face stability and considers a shield tunnelling under compressed air. In this case, the applied pressure is uniformly distributed on the tunnel face. The aim of the face stability analysis is to ensure safety against soil collapse and blowout in front of the tunnel face. A soil collapse occurs if the applied face pressure is not sufficient to prevent the movement of the soil mass towards the tunnel. On the other hand, a blowout appears when the applied face pressure is high enough to 'push' the soil towards the ground surface. It is desirable to assess both the collapse and the blowout face pressures and, thus, determine the acceptable range of air pressure to prevent both kinds of failure.

The study of the face stability of circular tunnels driven by pressurized shields has been investigated by several authors in literature. Some authors have considered a purely cohesive soil ([1-9] among others). In this case, it is usually considered that the stability of the tunnel face is governed by the so-called load factor $\mathrm{N}$, which was first defined in [1]. This load factor is given by $N=\left(\sigma_{s}+\gamma \cdot H-\sigma_{t}\right) / c_{u}$ where $\sigma_{s}$ is the possible surcharge loading acting on the ground surface, $\sigma_{t}$ is

*Correspondence to: Guilhem Mollon, Grenoble-INP, UJF-Grenoble 1, CNRS UMR 5521, 3SR, Grenoble F-38041, France.

†E-mail: guilhem.mollon@gmail.com 
the uniform pressure applied on the tunnel face, $\mathrm{H}$ is the depth of the tunnel axis, $\gamma$ is the soil unit weight and $c_{u}$ is the soil undrained cohesion. For the case of a frictional soil (with or without cohesion), some authors have performed experimental tests [10-13]. Others [14-25] have performed analytical or numerical approaches.

This paper focuses on the face stability analysis in the case of a purely cohesive soil. The kinematical theorem of limit analysis is chosen for this study because this approach is known to provide a rigorous upper bound of the critical load of a system. In case of collapse of a tunnel face, the critical face pressure $\sigma_{\mathrm{c}}$ is a 'resisting load' to failure, and therefore, the kinematical theorem provides an upper bound of $-\sigma_{\mathrm{c}}$ (i.e. a lower bound of $\sigma_{\mathrm{c}}$ ). On the other hand, the critical face pressure $\sigma_{\mathrm{b}}$ in the case of a face blowout is acting in the same direction as the movement and should be considered as an actual load. Therefore, the kinematical theorem provides a rigorous upper bound of $\sigma_{\mathrm{b}}$.

For the computation of the collapse and blowout pressures of purely cohesive soils in the framework of the kinematical approach in limit analysis, a simple and intuitive approach would be to adapt the rigid block failure mechanisms that already exist for frictional and cohesive soils to the case of a purely cohesive soil. Notice that the rigid block mechanisms were first introduced by [3] and [16], and then, several improvements were provided by [22, 24, 25] among others. The rigid-block failure mechanisms are either translational or rotational. The shape of these blocks is constrained by the normality condition, which implies that each velocity discontinuity should make an angle $\varphi$ with the corresponding velocity discontinuity surface, $\varphi$ being the internal friction angle of the soil. In the case of non-frictional soils, the shape of the failure mechanisms is still defined by the same normality condition but with $\varphi=0$. Figure 1 shows three typical failure mechanisms obtained by $[22,24,25]$ when imposing $\varphi=0$ in the analytical equations (i.e. for the case of a purely cohesive soil). Notice that, for purely cohesive soils, the collapse and blowout failure mechanisms based on rigid block mechanisms lead to the same critical surface even if the two velocity fields have opposite signs. This is a shortcoming as will be seen in the next section. On the other hand, Mollon et al. ([24] and [25]) have shown that the translational and rotational failure mechanisms composed of rigid blocks lead to relevant results in the case of frictional soils but may appear unrealistic for purely cohesive soils. A significant discrepancy exists between the results of these mechanisms and those obtained from centrifuge tests by [5]. To emphasize this statement, a picture of the collapse of a tunnel face in undrained clays as obtained by Schofield [4] using centrifuge testing is provided in Figure 2. From this picture, it clearly appears that the face collapse does not involve the motion of rigid blocks, but rather a continuous deformation of the soil. There is no localized shear band, and the motion of the soil mass appears more close to a 'flow' than a rigid movement towards the tunnel face.

To solve these issues, a numerical model is first presented hereafter. Its aim is to accurately assess the critical collapse and blowout pressures without any a priori assumption concerning the shape of the failure mechanisms. This model is implemented in the commercial code FLAC ${ }^{3 \mathrm{D}}$ and is described in Section 2. It will serve as a reference to explore suitable limit analysis velocity fields for purely cohesive soils in both

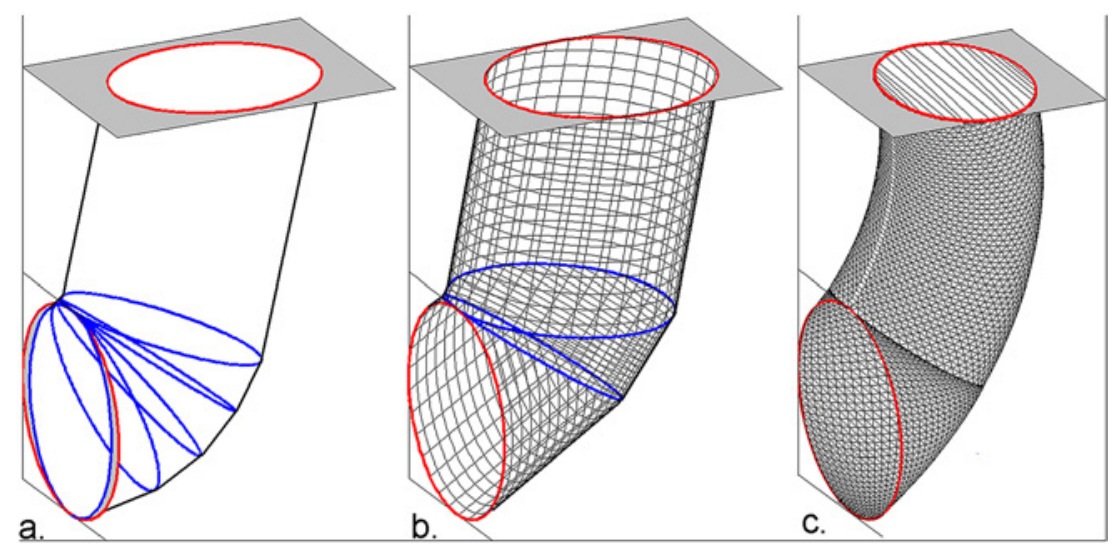

Figure 1. a, b and c: Rigid block failure mechanisms provided respectively by $[22,24,25]$ for $C / D=1$ when imposing $\varphi=0^{\circ}$. 


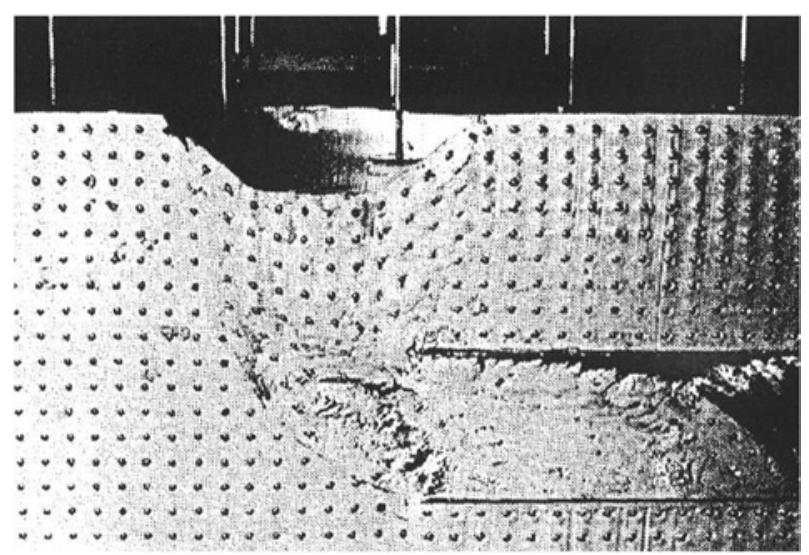

Figure 2. Picture of a face collapse in undrained clays as obtained by centrifuge experiment (provided by Schofield [4]).

cases of collapse and blowout. These velocity fields are described in some details in Section 3. Finally, Section 4 is devoted to a validation of the proposed velocity fields by comparison of their results with those of the numerical model described in Section 2. The paper ends with a conclusion.

\section{NUMERICAL MODEL USING FLAC ${ }^{3 D}$}

The numerical simulations presented in this study make use of the three-dimensional (3D) numerical model shown in Figure 3. These simulations are based on FLAC $^{3 \mathrm{D}}$ software (Fast Lagrangian Analysis of Continua [26]). This software is a commercially available 3D finite difference code. A key parameter used in the software is the so-called 'unbalanced force ratio'. It is defined at each calculation step (or cycle) as the average unbalanced mechanical force for all the grid points in the system divided by the average applied mechanical force for all these grid points. The system may be stable (in a steady state of static equilibrium) or unstable (in a steady state of plastic flow). A steady state of static equilibrium is one for which (i) a state of static equilibrium is achieved in the soilstructure system due to given loads with constant values of the soil displacement (i.e. vanishing values of the velocity) as the number of cycles increases and (ii) the unbalanced force ratio becomes smaller than a prescribed tolerance (e.g. $10^{-5}$ as suggested in FLAC ${ }^{3 \mathrm{D}}$ software). On the other hand, a steady state of plastic flow is one for which soil failure is achieved. In this case, although the unbalanced force ratio decreases as the number of cycles increases, this ratio does not tend to zero

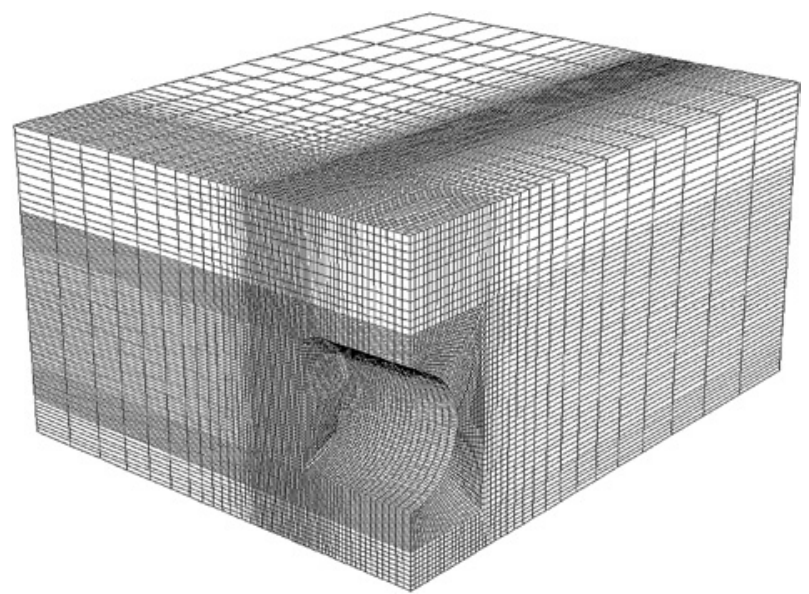

Figure 3. Numerical model for the analysis of face collapse and blowout. 
but attains a quasi-constant value. This value is usually higher than the one corresponding to the steady state of static equilibrium, but can be very small and still lead to infinite displacements, that is, to failure.

The dimensions of the 3D numerical model were $50 \times 40 \times 26 \mathrm{~m}$ in the transversal, longitudinal, and vertical directions, for a tunnel diameter $D=10 \mathrm{~m}$ and a cover to depth ratio $C / D=1$ (see Figure 3). The model was composed of 215,000 zones ('zone' is the FLAC ${ }^{3 \mathrm{D}}$ terminology for each discretized element). The tunnel face was composed of 800 zones. To study a purely cohesive soil, the soil was assigned an elastic perfectly plastic constitutive model based on Tresca failure criterion (which is identical to a Mohr-Coulomb criterion with $\varphi=0^{\circ}$ ). This corresponds to an undrained condition of a clayey soil where the soil plastic deformation takes place without any volume change. The elastic properties adopted in the analysis were $\mathrm{E}=240 \mathrm{MPa}$ and $v=0.49$. Notice that the elastic properties do not have any significant impact on the critical pressures. For this reason, a very high value of $\mathrm{E}$ was chosen because it increases the computation speed. The cylindrical lining of the tunnel was modelled as perfectly rigid.

The fastest method for the determination of the critical collapse or blowout pressure would be a strain-controlled method, but it is not suitable for the stability analysis of tunnels because it assumes that the deflected shape of the tunnel face is known. This shape is not known a priori, and any assumption (such as uniform or parabolic deflection) may lead to errors in the determination of the critical pressures. Thus, a stress-controlled method is used herein. It is illustrated in this section in the case of collapse; its application to the blowout case being straightforward.

An intuitive and simple approach in the stress-controlled method would consist in successively applying decreasingly prescribed uniform pressures on the tunnel face and searching for each applied pressure the static equilibrium until failure occurs. Of course, this requires a significant number of numerical simulations to obtain a satisfactory value of the critical collapse pressure. This makes the method very time consuming. A more efficient approach called the bisection method is suggested in this paper. This method allows the critical collapse pressure of the tunnel face to be determined with a much smaller computation time within an accuracy of $0.1 \mathrm{kPa}$. It can be described as follows:

- Define an initial lower bracket of the tunnel pressure: this pressure is any trial pressure for which the system is unstable. From a computational point of view, the system is considered as unstable if the tunnel face extrusion continues to increase (the velocity of this extrusion remaining almost constant) after 150000 computation cycles. Notice that the unbalanced force ratio adopted in this paper was equal to $10^{-7}$ because the value of $10^{-5}$ suggested in FLAC $^{3 \mathrm{D}}$ software was shown not to give an optimal solution in the present case. The choice of 150000 for the number of cycles has been determined after several trials; it is large enough to ensure that the system will never be stable if it is still unstable after running these 150000 cycles.

- Define an initial upper bracket of the tunnel pressure: this pressure is any trial pressure for which the system is stable. The system is considered as stable when the unbalanced force ratio drops under $10^{-7}$ before 150000 computation cycles.

- A new value, midway between the upper and lower brackets, is tested. If the system is stable for this midway value, the upper bracket is replaced by this trial pressure. If the system does not reach equilibrium, the lower bracket is then replaced by the midway value.

- The previous step is repeated until the difference between the upper and lower brackets is less than a prescribed tolerance, namely, $0.1 \mathrm{kPa}$ in this study. Because the width of the interval is divided by two at each step, a convenient method might be to use a first interval with a width equal to $n$ times $0.1 \mathrm{kPa}, n$ being a power of two.

The average computation time depends on the width of the first interval. For an interval width equal to $12.8 \mathrm{kPa}$, it is about 50 hours on a quad-core CPU. The numerical results of the critical collapse and blowout pressures obtained with this model are provided later in this paper. However, the shapes of the velocity fields obtained numerically are provided in Figure 4, for the collapse and blowout. Contrarily to the case of frictional soils (see [24] and [25]), it clearly appears that none of these failures involve rigid-block movement. Indeed, it appears that the failure propagates from the tunnel face to the ground surface, involving a continuous deformation of the soil mass. The point of maximum 

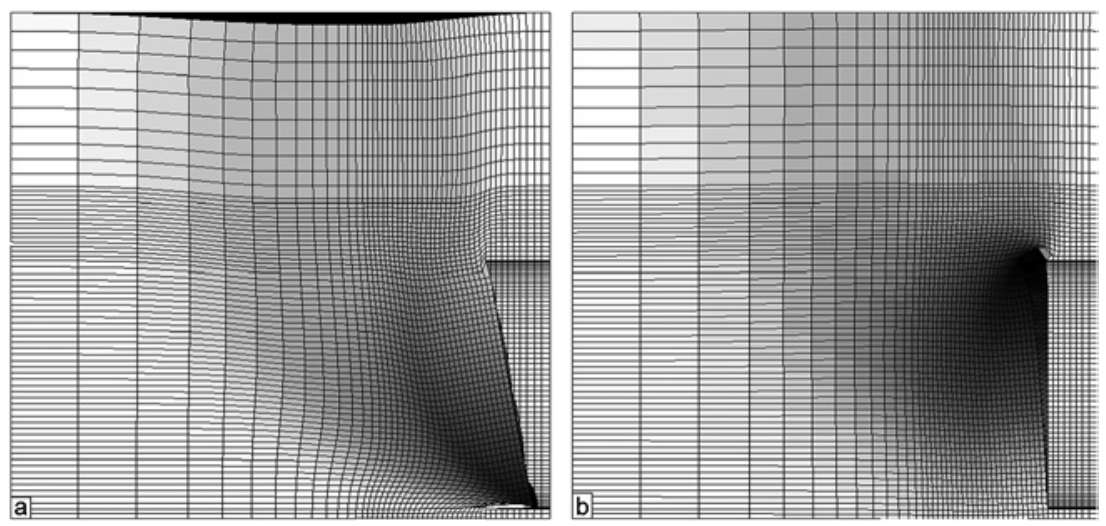

Figure 4. Layout of the a) collapse and b) blowout of the tunnel face as provided by the numerical model.

velocity is located at the invert of the tunnel face in the collapse case and at the crown of the tunnel face in the blowout case. The qualitative results of the numerical model are consistent with the experimental observations (as provided in Figure 2 for example) and show that a velocity field based on rigid blocks is not suitable for the face failure in purely cohesive soils. However, the time cost of this numerical model does not make it a convenient tool for practitioners. For this reason, the next section is devoted to the description of two new velocity fields, which are inspired from the qualitative results of the numerical model. These velocity fields are used in the framework of the kinematic theorem of limit analysis to obtain estimates of the critical pressures of collapse and blowout at a limited time cost.

\section{LIMIT ANALYSIS MODELS}

A tunnel of diameter $\mathrm{D}$ excavated under a cover depth $\mathrm{C}$ in a purely cohesive soil is considered in this study. The numerical results of Figure 4 have shown that failure mechanisms involving movement of rigid blocks (such as the ones presented in Figure 1) are not relevant for the collapse and the blowout of a tunnel face in a purely cohesive soil. To explore suitable velocity fields, one has to consider failures involving continuous deformation of the soil mass between the tunnel face and the ground surface. Within this framework, the most relevant approaches were proposed by [8] and [9]. Their methods make use of kinematically admissible continuous velocity fields based on elastic deformation fields. The numerical results obtained by these methods have shown a small improvement of the best existing bounds of the critical pressures. This is probably related to the fact that the chosen velocity fields were not perfectly adequate. However, the concept of continuous velocity fields is promising and deserves more investigation. This is the aim of the M1 and M2 velocity fields developed in this paper. Only the collapse case of the tunnel face is presented in details; the case of blowout is only briefly described because it is straightforward. It should be emphasized here that, although the M1 velocity field assumes a maximal velocity at the centre of the tunnel face and not at the invert of this face (as was observed in the numerical simulations), the M2 velocity field is an extension of M1, and it aims at removing this shortcoming as it will be seen in the following sections.

\subsection{M1 Velocity field}

3.1.1. Geometry. As shown in Figure 5, the external envelope of the M1 velocity field is a torus of centre $\mathrm{O}$. This toric shape was chosen to roughly represent with a simple geometrical object the envelope of the plastically deformed region of the soil mass as it was observed in numerical simulations. The section of this torus is a circle of variable radius $R(\beta)$ whose centre is located at a distance $R_{\mathrm{f}}$ from the centre of the torus, where $R_{\mathrm{f}}=C+D / 2$. The radius $R(\beta)$ of the circular section linearly increases from $R_{\mathrm{i}}=D / 2$ (at the tunnel face) to $R_{\mathrm{f}}$ (at the ground surface) as follows: 

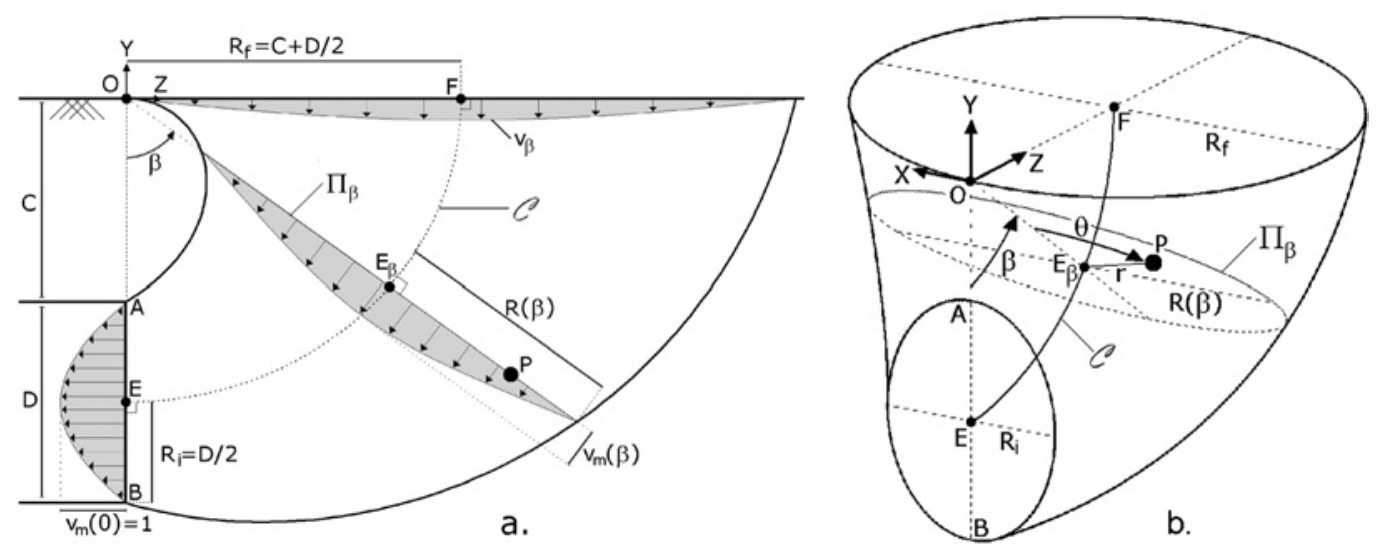

Figure 5. Layout of the M1 velocity field: a. Cross section in the plane of symmetry of the tunnel; b. threedimensional view.

$$
R(\beta)=R_{i}+\beta\left(R_{f}-R_{i}\right) /(\pi / 2) \quad \text { with } \quad 0 \leqslant \beta \leqslant \pi / 2
$$

In the toric system of axis defined by the circle $\mathfrak{C}$ of centre $O$ and radius $R_{\mathrm{f}}$, any point of the space may be expressed in terms of the angle $\beta$ (angle between the plane $\Pi_{\beta}$ and the plane of the tunnel face) and the polar coordinates $(r, \theta)$ in the plane $\Pi_{\beta}$. These coordinates are defined with respect to point $E_{\beta}$ located at the intersection between the plane $\Pi_{\beta}$ and the circle $\mathfrak{C}$ of centre $O$ and radius $R_{\mathrm{f}}$.

3.1.2. Velocities. As shown in Figure 6, the velocity vector of a given point $P$ whose toric coordinates are $(\beta, r, \theta)$ may be described by three components: (i) $v_{\beta}$ is the 'axial' component parallel to circle $\mathfrak{C}$ and pointing towards the tunnel face; (ii) $v_{\mathrm{r}}$ is the radial component located in the plane $\Pi_{\beta}$ and pointing towards $E_{\beta}$; and (iii) $v_{\theta}$ is the orthoradial component (i.e. orthogonal to the radial component and located in the plane $\Pi_{\beta}$ ).

The present M1 velocity field is based on the two following assumptions:

1. The profile of the axial component $v_{\beta}$ in any plane $\Pi_{\beta}$ is assumed to be symmetrical and to follow a parabolic distribution (cf. Figure 5). For a given point $(\beta, r, \theta)$, this axial component is given by

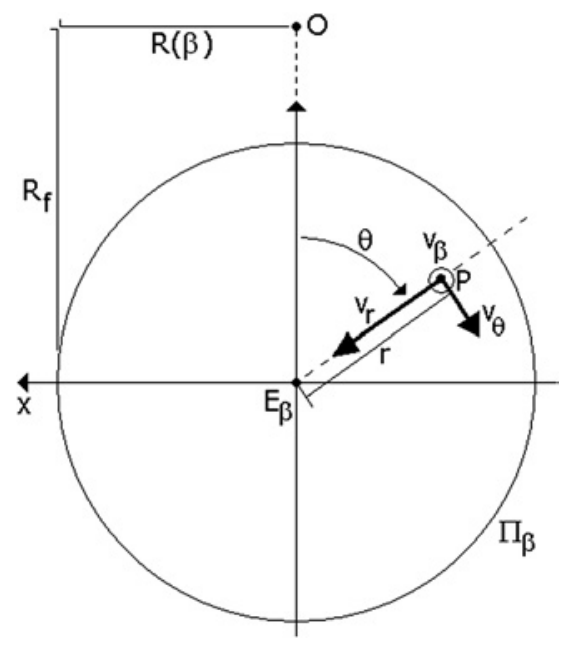

Figure 6. Components of the velocity field in a plane $\Pi_{\beta}$. 


$$
\left.v_{\beta}(\beta, r, \theta)=v_{m}(\beta) \cdot f(r, \beta)=v_{m}(\beta) \cdot 1-\frac{r^{2}}{[R(\beta)]^{2}}\right)
$$

The parabolic profile is imposed through the function $f(r, \beta)$, and the normalizing function $v_{m}(\beta)=$ $\left(\frac{R_{i}^{2}}{[R(\beta)]^{2}}\right)$ ensures that the velocity flux across any plane $\Pi_{\beta}$ is constant whatever is the angle $\beta$ (for more details, refer to Appendix A). As can be seen from Equation (2), $v_{\beta}$ does not depend on $\theta$ (i.e. there is a radial symmetry of $v_{\beta}$ in any plane $\Pi_{\beta}$ ), and $v_{\beta}$ is equal to zero on the lateral envelope of the velocity field.

2. The orthoradial component $v_{\theta}$ of the velocity vector is assumed to be equal to zero for all the $\Pi_{\beta}$ planes.

From the two above assumptions on the axial and orthoradial components of the velocity vector, one can compute the radial component $v_{\mathrm{r}}$ in any point of the velocity field using the normality condition for a non-frictional soil. The normality condition implies that any plastic deformation may occur without any change of volume. This condition may be enforced by using the following equation:

$$
\operatorname{div}(\dot{\varepsilon})=0
$$

Because the analytical expression of $\mathrm{v}_{\beta}$ is quite complex, the computation of $\mathrm{v}_{\mathrm{r}}$ is performed numerically using an explicit finite difference discretization scheme that was developed in the commercially available Matlab environment, as is shown in the following section. It should be remembered herein that only the orthoradial component of the velocity was assumed to be equal to zero everywhere in any plane $\Pi_{\beta}$. The radial velocity was not assumed to be equal to zero, neither in the tunnel face nor in any other $\Pi_{\beta}$ plane. However, this component (as well as the two others) should be equal to zero on the external envelope of the velocity field in order to avoid any velocity jump on this envelope. Moreover, because of the toric geometry of the problem and contrarily to the axial component of the velocity field, the radial component is not expected to be symmetrical with respect to the central circle $\mathfrak{C}$ of the toric system of coordinates.

3.1.3. Numerical discretization of the M1 velocity field for the computation of the radial component of the velocity vector. Although the plastically deformed soil mass involved by the velocity field is bounded by a torus with a circular cross section of linearly increasing radius (Figure 7a), the mesh used for the discretization of the velocity field is bigger and involves a quarter of a torus, which has a constant radius $R_{\mathrm{f}}$ (i.e. a constant circular cross section) as shown in Figure (7b). Figure (7b) allows one to see not only the mesh but also the position of the plastically deformed soil mass with

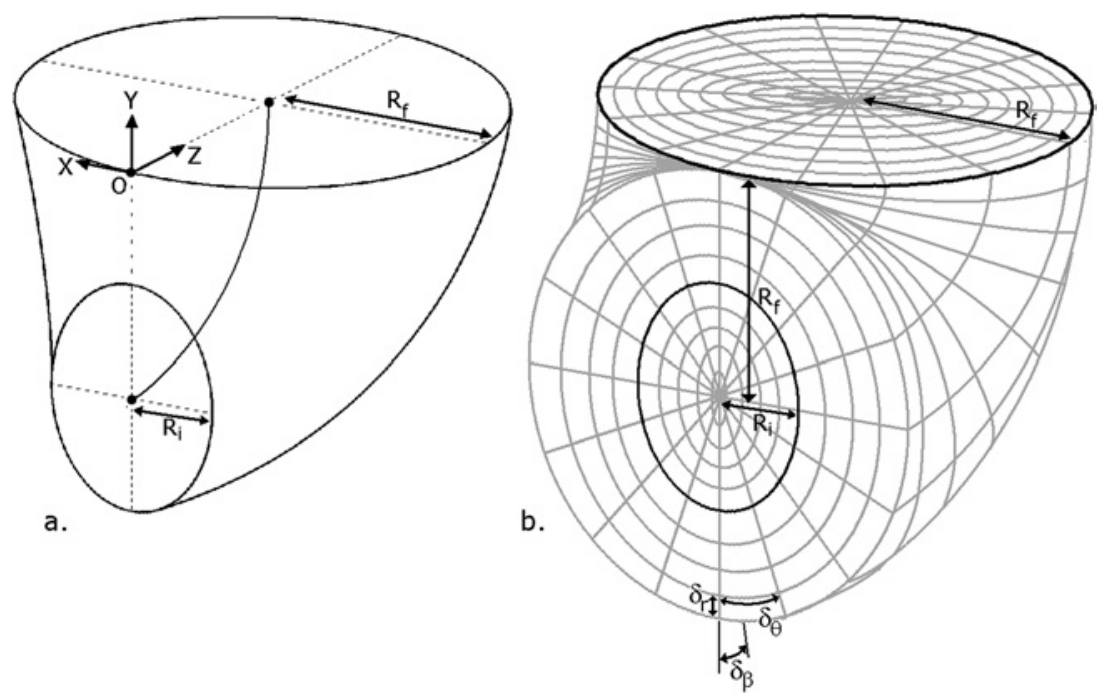

Figure 7. a. Volume of the plastically deformed soil mass; b. Mesh used in the analysis. 
respect to this mesh. The size of each zone (or element) of the mesh is defined by three parameters $\delta_{\mathrm{r}}$, $\delta_{\beta}$ and $\delta_{\theta}$ (Figure $7 \mathrm{~b}$ ) expressed by

$$
\left\{\begin{array}{c}
\delta_{r}=R_{f} / N_{r} \\
\delta_{\beta}=\pi /\left(2 \cdot N_{\beta}\right) \\
\delta_{\theta}=2 \pi / N_{\theta}
\end{array}\right.
$$

where $N_{\mathrm{r}}, N_{\beta}$ and $N_{\theta}$ are the numbers of subdivisions in the three directions $r, \beta$ and $\theta$. A typical zone with a central point $P(\beta, r, \theta)$ is represented in Figure 8 . It is bounded by six curved facets $S_{1}, S_{2}, S_{3}$, $S_{1}, S_{2}$ and $S_{3}$. If the discretization is fine enough (i.e. if the parameters $\delta_{\mathrm{r}}, \delta_{\beta}$ and $\delta_{\theta}$ are small enough), one may consider that these facets are planar. Notice that the mesh provided in Figure $7 \mathrm{~b}$ is very coarse (the discretization parameters were $N_{\mathrm{r}}=9, N_{\beta}=8$ and $N_{\theta}=16$ ) to make it clearer but that a much finer mesh was used for the computations $\left(N_{\mathrm{r}}=200, N_{\beta}=90\right.$ and $\left.N_{\theta}=90\right)$.

The axial component $v_{\beta}$ of the velocity vector is first computed for each zone of the discretized domain using Equation (2). This component is considered equal to zero in all the mesh elements that are outside the envelope of the velocity field. Then, Equation (3) is used to compute the radial component of the velocity for the different elements as will be explained below.

Notice that Equation (3) corresponds to a plastic deformation at constant volume. Thus, it may be rewritten for each mesh element as follows:

$$
\sum_{i=1}^{3} \vec{v} \cdot \overrightarrow{n_{i}} \cdot S_{i}+\sum_{i=1}^{3} \vec{v} \cdot \overrightarrow{n_{i}^{\prime}} \cdot S_{i}^{\prime}=0
$$

In this expression, $\overrightarrow{n_{i}}$ represents the normal vector to facet $S_{\mathrm{i}}$ (pointing outwards), and $\overrightarrow{n_{i}^{\prime}}$ represents the normal vector to facet $S_{\mathrm{i}}$ ' (pointing outwards). By considering that the velocities are quasi-uniform on the discretized facets, Equation (5) may be re-written as follows:

$$
v_{r}\left(P_{1}\right) \cdot S_{1}-v_{r}\left(P_{1}^{\prime}\right) \cdot S_{1}^{\prime}+v_{\theta}\left(P_{2}\right) \cdot S_{2}-v_{\theta}\left(P_{2}^{\prime}\right) \cdot S_{2}^{\prime}+v_{\beta}\left(P_{3}\right) \cdot S_{3}-v_{\beta}\left(P_{3}^{\prime}\right) \cdot S_{3}^{\prime}=0
$$

Details on the computation of the facets' surfaces and the coordinates of the different points $P_{\mathrm{i}}$ and $P_{\mathrm{i}}{ }^{\prime}$ are given in Appendix B. It should be remembered here that the orthoradial component of the

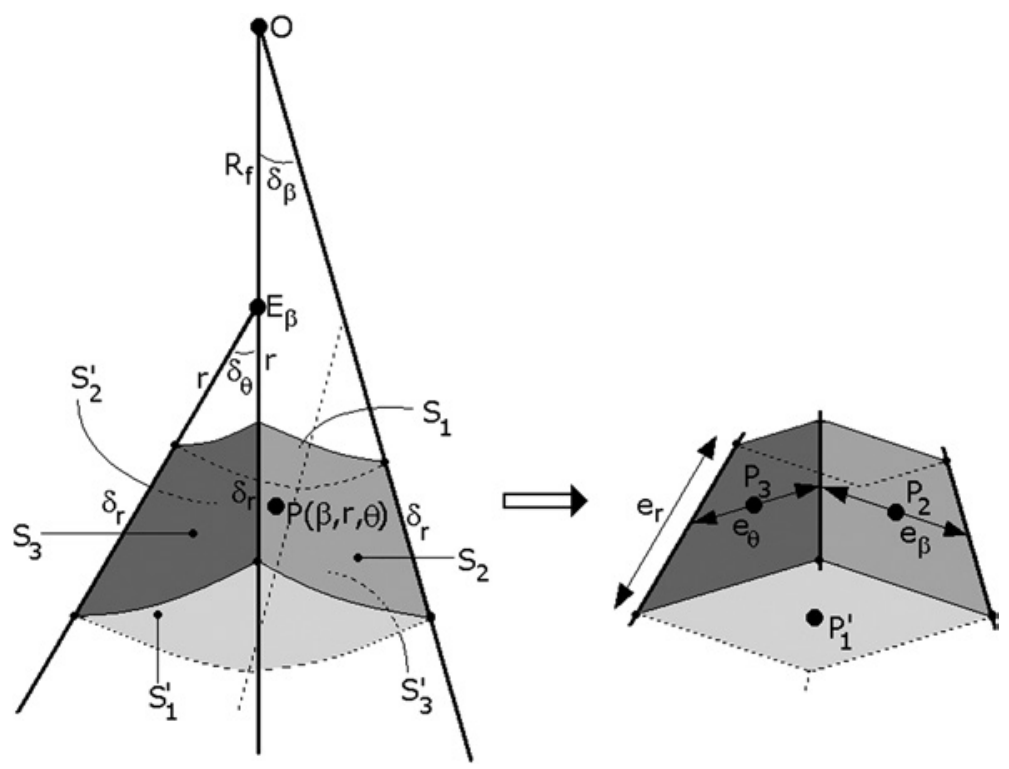

Figure 8. Detail of an element of the discretized mesh. 
velocity $v_{\theta}$ was assumed to be null, and the axial component $v_{\beta}$ at points $P_{3}$ and $P_{3}$ ' may be computed using Equation (2). Thus, by using Equation B1 of Appendix B, which states that $S_{3}=S_{3}$, Equation (6) may be rewritten as follows:

$$
v_{r}\left(P_{1}^{\prime}\right)=\frac{v_{r}\left(P_{1}\right) \cdot S_{1}+\left[v_{\beta}\left(P_{3}\right)-v_{\beta}\left(P_{3}^{\prime}\right)\right] \cdot S_{3}}{S_{1}^{\prime}}
$$

Equation (7) can be used for the computation of the radial velocity at point $P_{1}{ }^{\prime}$ as long as this velocity is known at point $P_{1}$. The radial velocity at any point $P_{1}$ of the mesh can thus be computed using the boundary condition, which assumes that $v_{\mathrm{r}}=0$ for $r=0$ (i.e. the radial component of the velocity is null on the circle $\left(\mathfrak{C}\right.$ ). The velocity component $v_{\mathrm{r}}$ at any point $P$ (at the centre of a mesh element) is given by the average between the ones at points $P_{1}$ and $P_{1}$ ' as follows:

$$
v_{r}(P)=\frac{v_{r}\left(P_{1}^{\prime}\right)+v_{r}\left(P_{1}\right)}{2}
$$

Figure 9 shows the distributions of the axial $\left(v_{\beta}\right)$ and radial $\left(v_{\mathrm{r}}\right)$ components of the velocity field computed by the method described above for $C=D=10 \mathrm{~m}$. These components are plotted in shades of grey (the white corresponding to a zero velocity) in several areas of the velocity field (at the tunnel face, on the outcropping surface, and in the symmetry plane of the tunnel, i.e. where $X=0$ ). It clearly appears that the radial component of the velocity is equal to zero on all the lateral surfaces of the velocity field. This result is interesting and is in conformity with the assumptions of the velocity field (i.e. there is no velocity discontinuity on the external envelope of this velocity field).

3.1.4. Work equation. The determination of the collapse pressure is based on the work equation. The rate of work of the external forces may be computed using the components of the velocity field in the different mesh elements. The rate of work of the soil weight for a given mesh element of volume $\delta V$ is given by

$$
\delta \dot{W}_{\gamma}=\vec{\gamma} \cdot \vec{v} \delta V=\gamma \cdot v_{Y} \cdot \delta V
$$

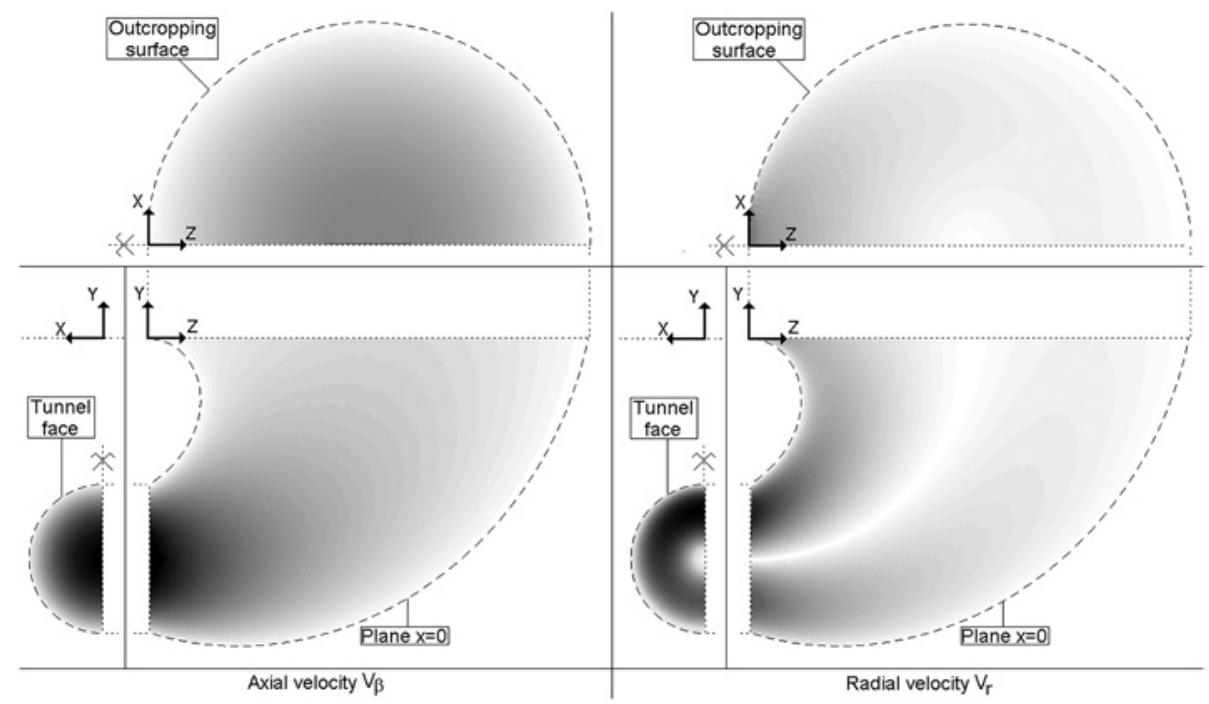

Figure 9. Axial and radial components of the M1 velocity field in several areas (tunnel face, ground surface, plane of symmetry $\mathrm{X}=0$ ). The greyscale represents the normalized value of the corresponding component, from 0 (white) to its maximum value (black). 
In this equation, the vertical component $v_{\mathrm{Y}}$ of the velocity field in the $(X, Y, Z)$ system of axis can be computed directly from $v_{\beta}$ and $v_{\mathrm{r}}$ as follows:

$$
v_{Y}=-v_{\beta} \cdot \sin \beta-v_{r} \cdot \cos \theta \cdot \cos \beta
$$

Thus, the total rate of work of the soil weight is given by

$$
\dot{W}_{\gamma}=\gamma \cdot \sum_{N_{\beta}, N_{r}, N_{\theta}}\left(v_{Y} \cdot \delta V\right)
$$

For a given mesh element belonging to the tunnel face, the rate of work of the tunnel face pressure is given by

$$
\delta \dot{W}_{\sigma c}=\overrightarrow{\sigma_{c}} \cdot \vec{v} S_{3}=-\sigma_{c} \cdot v_{\beta} \cdot S_{3}
$$

For a constant tunnel pressure as is the case of an air-pressurized tunnel, the total rate of work of the tunnel face pressure is given by

$$
\dot{W}_{\sigma c}=-\sigma_{c} \cdot \sum_{N_{r}, N_{\theta}, \beta=0}\left(v_{\beta} \cdot S_{3}\right)
$$

The total rate of work of a possible uniform surcharge acting on the ground surface is given by

$$
\dot{W}_{\sigma s}=\sigma_{s} \cdot \sum_{N_{r}, N_{\theta}, \beta=\pi / 2}\left(v_{\beta} \cdot S_{3}^{\prime}\right)
$$

The rate of energy dissipation of a soil element undergoing a general plastic deformation may be written as follows (cf. [27]):

$$
\delta \dot{D}=c_{u} \cdot\left(2 \cdot \max \left(\left|\dot{\varepsilon}_{i}\right|\right) \cdot \delta V\right)
$$

where $\max \left(\left|\ddot{\varepsilon}_{i}\right|\right)$ is the maximal absolute value of the principal strain rate components $\dot{\varepsilon}_{i}$ where $i=1,2,3$. Appendix $\mathrm{C}$ provides the method of computation of the strain rate tensor for a given element of the mesh. Matlab software was used to transform this tensor into the principal space. Then, Equation (15) was applied for the computation of the rate of energy dissipation of this soil element. The total rate of energy dissipation in the M1 velocity field was obtained by summation of the elementary energy dissipation for all the elements of the mesh as follows:

$$
\dot{D}=c_{u} \cdot \sum_{N_{\beta}, N_{r}, N_{\theta}}\left(2 \cdot \max \left(\left|\dot{\varepsilon}_{i}\right|\right) \cdot \delta V\right)
$$

By equating the total rate of work of the external forces to the total rate of internal energy dissipation, one obtains

$$
\dot{W}_{\gamma}+\dot{W}_{\sigma c}+\dot{W}_{\sigma s}=\dot{D}
$$

After some simplifications, Equation (17) leads to the following equation, which gives a rigorous lower bound of the critical collapse pressure $\sigma_{\mathrm{c}}$ : 


$$
\sigma_{c}=\gamma \cdot D \cdot N_{\gamma}-c_{u} \cdot N_{c}+\sigma_{s} \cdot N_{s}
$$

This equation shows that (as for the classical problem of bearing capacity of foundations) the collapse pressure includes three different components related to soil weight, soil cohesion and surcharge loading. The corresponding $\mathrm{N}_{\gamma}, \mathrm{N}_{\mathrm{c}}$ and $\mathrm{N}_{\mathrm{s}}$ coefficients are dimensionless parameters. They are given by

$$
\begin{gathered}
N_{\gamma}=\frac{\sum_{N_{\beta}, N_{r}, N_{\theta}}\left(v_{Y} \cdot \delta V\right)}{D \cdot \sum_{N_{r}, N_{\theta}, \beta=0}\left(v_{\beta} \cdot S_{3}\right)} \\
N_{c}=\frac{\sum_{N_{\beta}, N_{r}, N_{\theta}}\left(2 \cdot \max \left(\left|\dot{\varepsilon}_{i}\right|\right) \cdot \delta V\right)}{\sum_{N_{r}, N_{\theta}, \beta=0}\left(v_{\beta} \cdot S_{3}\right)} \\
N_{S}=\frac{\sum_{N_{r}, N_{\theta}, \beta=\pi / 2}\left(v_{\beta} \cdot S_{3}^{\prime}\right)}{\sum_{N_{r}, N_{\theta}, \beta=0}\left(v_{\beta} \cdot S_{3}\right)}
\end{gathered}
$$

It should be remembered here that the velocity field described above allows the computation of the critical collapse pressure $\sigma_{\mathrm{c}}$. However, in some cases of tunnelling at very shallow depths and in weak soils, it may be interesting to compute also the critical blowout pressure $\sigma_{\mathrm{b}}$, which corresponds to the maximal retaining pressure that one can apply to the tunnel face without producing a blowout to the ground surface. Because the collapse velocity field presented above is kinematically admissible, the velocity field, which is strictly opposite, is kinematically admissible as well and may be used for a kinematical analysis of the blowout phenomenon. By adopting the velocity field provided in Figure 9 for the blowout case, a rigorous upper-bound of the blowout pressure is given by an equation that is similar to Equation (18) except that the $N_{\mathrm{c}}$ coefficient is now given by

$$
N_{c}=-\frac{\sum_{N_{\beta}, N_{r}, N_{\theta}}\left(2 \cdot \max \left(\left|\dot{\varepsilon}_{i}\right|\right) \cdot \delta V\right)}{\sum_{N_{r}, N_{\theta}, \beta=0}\left(v_{\beta} \cdot S_{3}\right)}
$$

Thus, in the case of blowout, the coefficients $N_{\gamma}$ and $N_{\mathrm{s}}$ are identical to the ones used in the collapse case and are given by Equations (19) and (21), respectively. However, the $N_{\mathrm{c}}$ coefficient of $\sigma_{\mathrm{b}}$ has an opposite sign with respect to the one given for $\sigma_{\mathrm{c}}$.

\subsection{M2 Velocity field}

As was mentioned previously, the M2 velocity field is an extension of the M1 velocity field. It aims at removing the shortcoming related to the maximal velocity at the centre of the tunnel face. This velocity field is presented in Figure 10. Contrarily to M1, it was assumed that the point of maximum axial velocity at the tunnel face in case of collapse is not located at the centre but downwards at a distance $L_{1}$ below this centre. A new circle $\mathfrak{C}$ of centre $O$ and new radius $R_{\mathrm{f}}$ was defined. This radius depends on $L_{1}$ (cf. Figure 10a) and is given by

$$
R_{f}=C+D / 2+L_{1}
$$

Similarly to the tunnel face, for an arbitrary plane $\Pi_{\beta}$, it was assumed that the axial component of the velocity vector is maximal at point $E_{\beta}$ (belonging to circle $\mathfrak{C}$ ) and decreases in a parabolic way to 


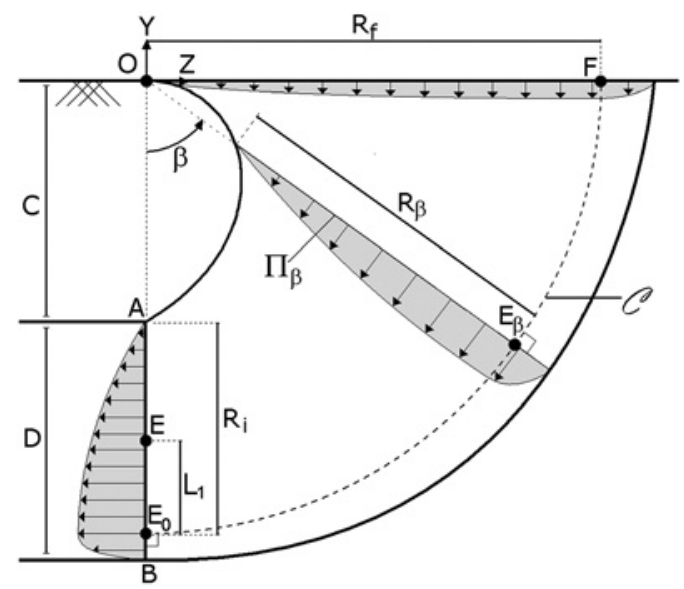

a

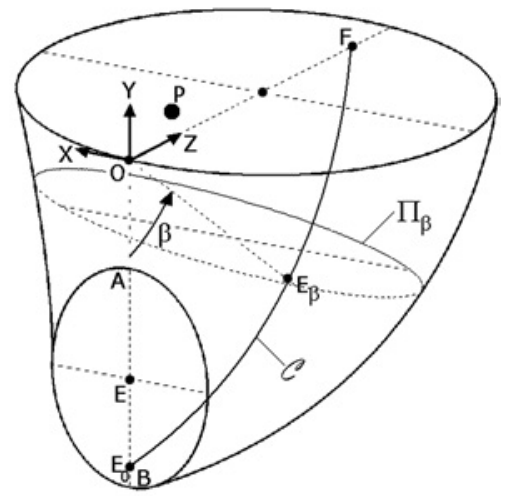

b

Figure 10. Layout of the M2 velocity field: a. Cross section in the plane of symmetry of the tunnel; b. threedimensional view.

vanish when reaching the circular contour of the envelope. Therefore, in a given plane $\Pi_{\beta}$, there is no more radial symmetry of the axial component of the velocity field as was the case in M1. A toric system of axis is defined in the same way as for the M1 velocity field except that its radius is defined by Equation (23). For a given point $(\beta, r, \theta)$, the axial component $v_{\beta}$ and the orthoradial component $v_{\theta}$ of the velocity vector are defined as follows:

$$
\left\{\begin{array}{l}
\left.v_{\beta}(\beta, r, \theta)=v_{m}(\beta) \cdot f(r, \beta, \theta)=v_{m}(\beta) \cdot 1-\frac{r^{2}}{\left[r_{\max }(\beta, \theta)\right]^{2}}\right) \\
v_{\theta}(\beta, r, \theta)=0
\end{array}\right.
$$

By comparing Equation (24) with Equation (2), one may observe that the term $r_{\max }(\beta, \theta)$ appears instead of $R(\beta)$. A graphical representation of this term is given in Figure 11 for both the plane $\Pi_{0}$ (i.e. the tunnel face) and an arbitrary plane $\Pi_{\beta}$. In a given plane $\Pi_{\beta}$ and for a given angle $\theta$, the term $r_{\max }$ represents the distance between point $E_{\beta}$ (belonging to circle $\mathfrak{C}$ ) and the circular contour of the envelope in this plane. This term is used to ensure that the axial velocity is equal to zero in any point of this envelope. However, notice that, because of the fact that $r_{\max }$ depends not only on $\beta$

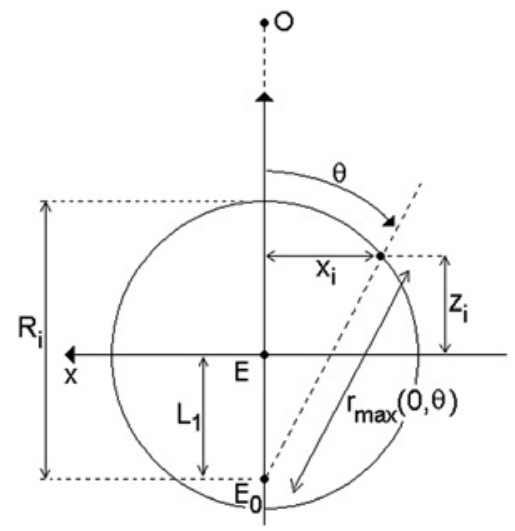

$\Pi_{0}$

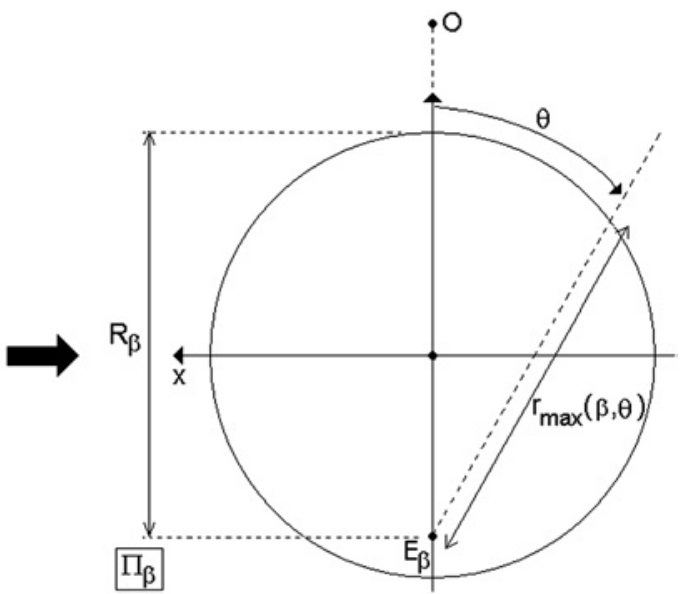

$\Pi_{\beta}$

Figure 11. Geometrical details of the M2 velocity fie $1 \mathrm{~d}$ in $\mathrm{p} 1$ a n e $\Pi_{0}$ (i.e. tunnel face) and plane $\Pi_{\beta}$ (arbitrary plane). 
but also on $\theta$ as well, the external envelope of the M2 velocity field is not a torus of circular cross section as was the case in M1. In fact, although the envelope of the M2 velocity field is defined by a series of circles, these circles do not form a torus of circular cross section because the curve joining the different centres of these circles is not circular. The computation of $r_{\max }(\beta, \theta)$ is detailed in Appendix D.

On the other hand, the term $v_{\mathrm{m}}(\beta)$ in Equation (24) is a normalizing term, which depends on the position of the plane $\Pi_{\beta}$. It is used to ensure that the velocity flux across any plane $\Pi_{\beta}$ is constant for the different values of $\beta$. The expression of $v_{m}(\beta)$ is more complex than the one of the M1 velocity field. Thus, it was not possible to obtain an analytical expression of the axial velocity $v_{\beta}$ as was done in the previous velocity field. A numerical method is therefore used to assess $v_{\beta}$ in any plane $\Pi_{\beta}$ as follows:

A toric discretized mesh is used. It is identical to the one used in M1 (cf. Figure 7), except that the new circle $\mathfrak{C}$ with the new radius $R_{\mathrm{f}}$ (Equation 23) is used when performing the discretization. For each element of an arbitrary plane $\Pi_{\beta}$ of the mesh, the following term is first computed:

$$
f(r, \beta, \theta)=1-\frac{r^{2}}{\left[r_{\max }(\beta, \theta)\right]^{2}}
$$

This term decreases from 1 (at $r=0$, i.e. on the circle $\mathfrak{C}$ ) to 0 (at $\mathrm{r}=r_{\max }$, i.e. on the external envelope of the velocity field) according to a parabolic distribution. Thus, Equation (25) represents the distribution of the axial component of the velocity field in the plane $\Pi_{\beta}$ as given in Equation (24), except that it is not 'normalized' by the term $v_{\mathrm{m}}(\beta)$ because this term is so far unspecified. The axial component $v_{\beta}$ of the velocity is then obtained by multiplying $f(r, \beta, \theta)$ by the normalizing term $v_{\mathrm{m}}(\beta)$. This term is obtained by assuming that the integration of $v_{\beta}$ over the $\Pi_{\beta}$ plane is constant and equal to one as follows:

$$
v_{m}(\beta)=\frac{1}{\iint_{\Pi_{\beta}} f(r, \beta, \theta) \cdot d S}
$$

The discretized form of this equation may be written as follows:

$$
v_{m}(\beta)=\frac{1}{\sum_{N_{r}} \sum_{N_{\theta}} f(r, \beta, \theta) \cdot \delta_{r} \cdot r \cdot \delta_{\theta}}
$$

As one may see, the computation of the axial component of the velocity field (through the use of Equation (24)) is made by first computing a non-normalized function $f(r, \beta, \theta)$ using Equation (25) and then by multiplying it by $v_{m}$ using Equation (27), i.e. by dividing it by the integral of the nonnormalized function $f(r, \beta, \theta)$ across the plane $\Pi_{\beta}$. This ensures that the integral of the axial velocity profile across any plane $\Pi_{\beta}$ is equal to 1 and, therefore, independent of $\beta$.

The knowledge of the components $v_{\beta}$ and $v_{\theta}$ in any point of the discretized mesh allows one to obtain the radial component $\mathrm{v}_{\mathrm{r}}$ by using Equations (7) and (8) that have been developed for the M1 velocity field. As for M1, a null radial velocity on the circle $\mathfrak{C}$ is assumed as a boundary condition. The main difficulty encountered was the existence of an important gradient of the axial component of the velocity field in the lower part of the tunnel face. This gradient may become infinite for $L_{1}=\mathrm{D} / 2$. Notice that the gradient of the axial velocity is directly linked to the fact that the point of maximal velocity at the tunnel face was moved downwards with respect to the M1 velocity field. Notice also that the simple method of discretization proposed in this paper is not able to deal with a velocity discontinuity and may lead to numerical errors if the velocity gradient is too large. A first solution would be to drastically refine the mesh, especially in the radial direction because this is the one with the largest gradients. However, this solution has the shortcoming of significantly increasing 
the computation time. An intermediate solution was chosen: the mesh was moderately refined in the area of interest with respect to the mesh of M1, and the following condition was arbitrarily adopted:

$$
L_{1}=\frac{2}{5} D
$$

This condition implies that the distance between the tunnel face invert and the point of maximal velocity is equal to one fifth of the tunnel radius. This induces a reduction of the radial gradient of the velocity without decreasing the accuracy of the numerical results. The axial and radial components of the velocity field obtained with this assumption are plotted in Figure 12. As for the M1 velocity field, the numerical method of determination of the radial component leads to a kinematically admissible velocity field without velocity discontinuity on the external envelope of the velocity field where all the velocity components are equal to zero. A rather good match is obtained when comparing visually this postulated velocity field with the one obtained experimentally by [4] and shown in Figure 2. Figure 13 presents the velocity profile at the tunnel face as provided by the M1 and M2 velocity fields and by the numerical model. M2 appears to provide a velocity profile that is much more consistent with the one obtained numerically. The coefficients $N_{\gamma}, N_{\mathrm{c}}$ and $N_{\mathrm{s}}$ of this velocity field can be computed using the same equations used for the M1 velocity field, that is, Equations (19), (20) and (21), respectively, but by employing the velocities determined from the M2 velocity field. On the other hand, the collapse pressure can be calculated using Equation (18) with the velocity components determined from the M2 velocity field.

As for M1, the M2 velocity field may also be extended to compute the critical pressure of blowout. The numerical results by FLAC ${ }^{3 \mathrm{D}}$ have shown that, in the case of blowout, the point with a maximum velocity is located in the upper part of the tunnel face. It was assumed that the point of maximum velocity is located at a distance $L_{1}$ (defined by Equation (28)) above the centre of the tunnel face. Moreover, the velocity field in the case of blowout should point upwards in the direction of the ground surface. With these assumptions, a rigorous upper-bound of the critical blowout pressure is provided by Equation (18) where the dimensionless coefficients $N_{\gamma}, N_{\mathrm{s}}$ and $N_{\mathrm{c}}$ are given by Equations (19), (21) and (22), respectively. It should be emphasized here that contrary to the case of M1, the values of the coefficients $N_{\gamma}$ and $N_{\mathrm{c}}$ are different in the cases of collapse and blowout. This

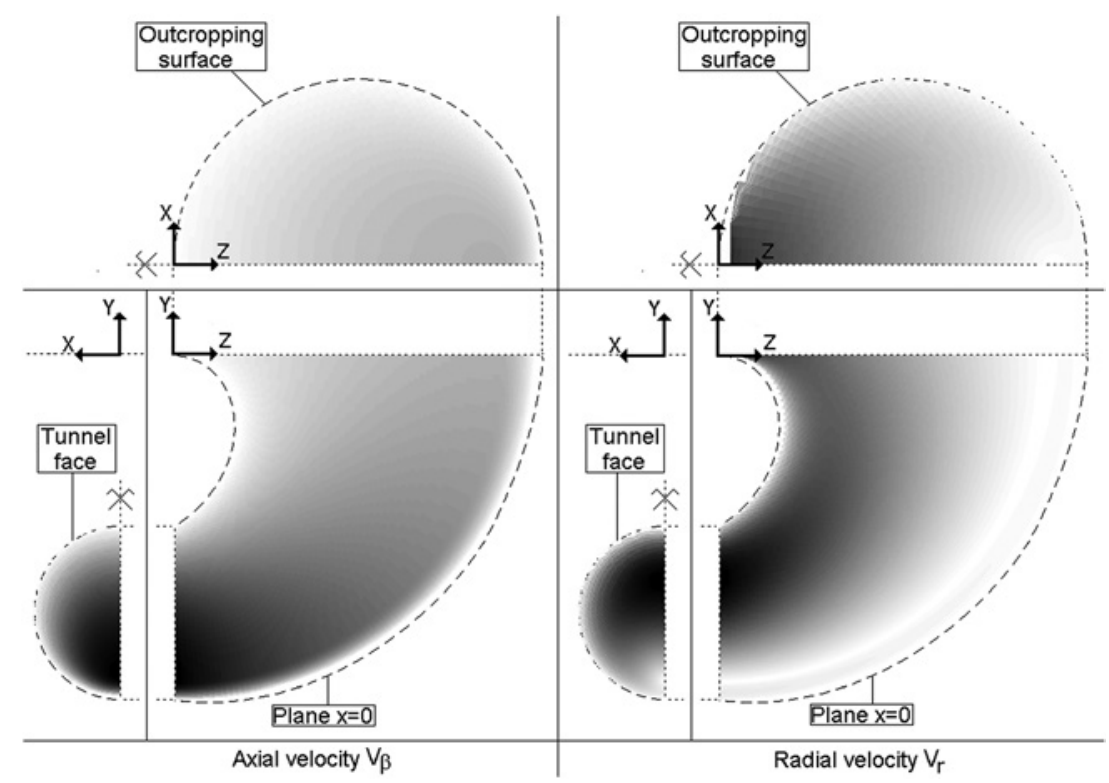

Figure 12. Axial and radial components of the M2 velocity field in several areas (tunnel face, ground surface, plane of symmetry $\mathrm{X}=0$ ). The greyscale represents the normalized value of the corresponding component, from 0 (white) to its maximum value (black). 


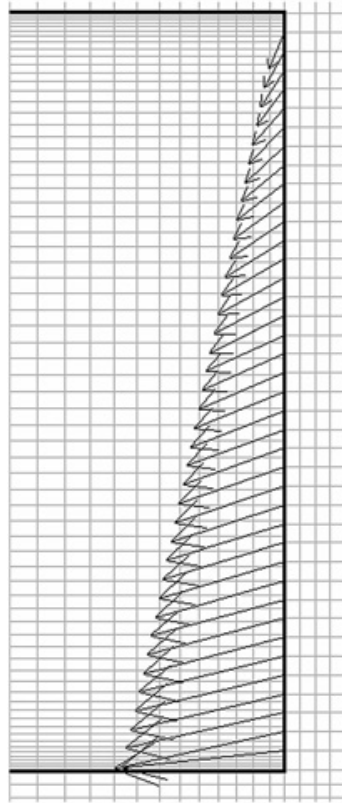

Numerical velocity field (Flac3D)

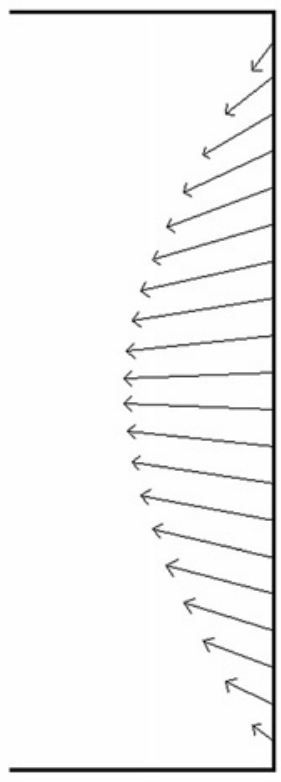

Analytical velocity field M1

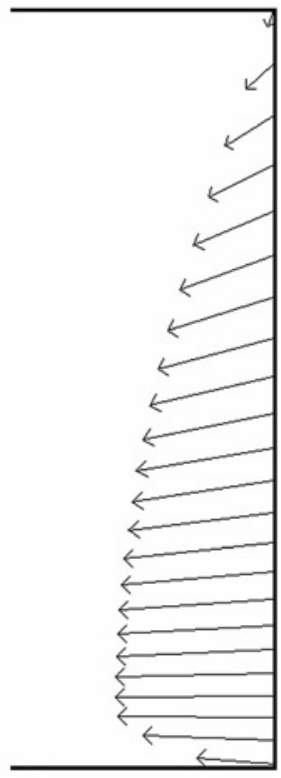

Analytical velocity field M2

Figure 13. Comparison between the velocity profiles at the tunnel face as provided by the numerical simulation using FLAC ${ }^{3 \mathrm{D}}$ and by M1 and M2 velocity fields.

is directly linked to the fact that the velocity fields of these two failure modes are not directly opposite because of the position of the point of maximum velocity which is either in the upper or lower part of the tunnel face.

\section{NUMERICAL RESULTS}

Two computer programs based on the M1 and M2 velocity fields were coded in Matlab to compute the critical pressures of collapse and blowout. The computation time is approximately equal to 20 seconds, using the four processors of a $2.4 \mathrm{GHz}$ quad-core CPU computer.

For purely cohesive soils, the traditional method used to check the stability of a tunnel face is based on the so-called load factor $N$ (cf. [1,3]). The definition of this factor is given by the following equation ( $H=C+D / 2$ being the depth of the tunnel axis):

$$
N=\left(\sigma_{s}+\gamma \cdot H-\sigma_{c}\right) / c_{u}
$$

In the more general case of a frictional and cohesive soil, the critical collapse pressure may be assessed by using the following equation:

$$
\sigma_{c}=\gamma \cdot D \cdot N_{\gamma}-c \cdot N_{c}+\sigma_{s} \cdot N_{s}
$$

By using Equations (29) and (30), one may deduce the $N_{\gamma}, N_{c}$ and $N_{s}$ coefficients of a purely cohesive soil as follows:

$$
N_{\gamma}=H / D=C / D+0.5
$$




$$
N_{c}=N
$$

$$
N_{s}=1
$$

Equation (31) states that the coefficient $N_{\gamma}$ related to the soil weight is equal to the H/D ratio. Thus, it is independent from the geometrical parameters of the failure mechanism and only depends on the geometry of the problem (tunnel diameter and depth). On the other hand, Equation (32) expresses the fact that the load factor used for purely cohesive soils is equal to the coefficient $N_{\mathrm{c}}$ employed in Equation (30) to characterize the effect of soil cohesion in the collapse pressure. Finally, Equation (33) may be explained by the fact that the soil plastic deformation occurs at constant volume. It may be justified mathematically by Equation (21), which shows that $N_{\mathrm{s}}$ is equal to the ratio between the velocity flux across the ground surface and the one across the tunnel face. Remember here that the flux of velocity across the tunnel face and the one across the ground surface are equal (see Appendix A for M1 and equation (26) for M2). Thus, $N_{\mathrm{s}}$ is equal to 1 for purely cohesive soils and the two pressure values $\sigma_{\mathrm{s}}$ (applied at the ground surface) and $\sigma_{\mathrm{c}}$ (applied at the tunnel face) may be directly taken into account by their difference $\left(\sigma_{\mathrm{c}}-\sigma_{\mathrm{s}}\right)$ in the case of a purely cohesive soil as may be seen from Equation (29).

The numerical results obtained by the M1 velocity field have confirmed the above cited observations about the $N_{\gamma}, N_{c}$ and $N_{s}$ coefficients and the load factor $\mathrm{N}$. These observations were also obtained with the rigid block mechanisms by Mollon et al. [22, 24, 25]. Consequently, for these mechanisms, the knowledge of $N$ is sufficient to obtain the critical pressures of collapse and blowout or to assess the stability of the tunnel face. The values of $N$ provided by several authors and by the present M1 velocity field are given in Figure 14 for different values of the ratio $C / D$. Because the kinematical theorem leads to an upper-bound of $N$, all the kinematical approaches have provided as expected higher values than the other approaches. The M1 velocity field is the one which provides the best (i.e. lowest) upper-bound for $C / D$ values larger than 2, and the failure mechanism by [24] provides the best upper-bounds for smaller covers. The static approach by [3] provides a lower-bound of $N$, which appears quite far from the best upper-bounds. With these bounds, the estimation of the actual value of $\mathrm{N}$ is not very accurate.

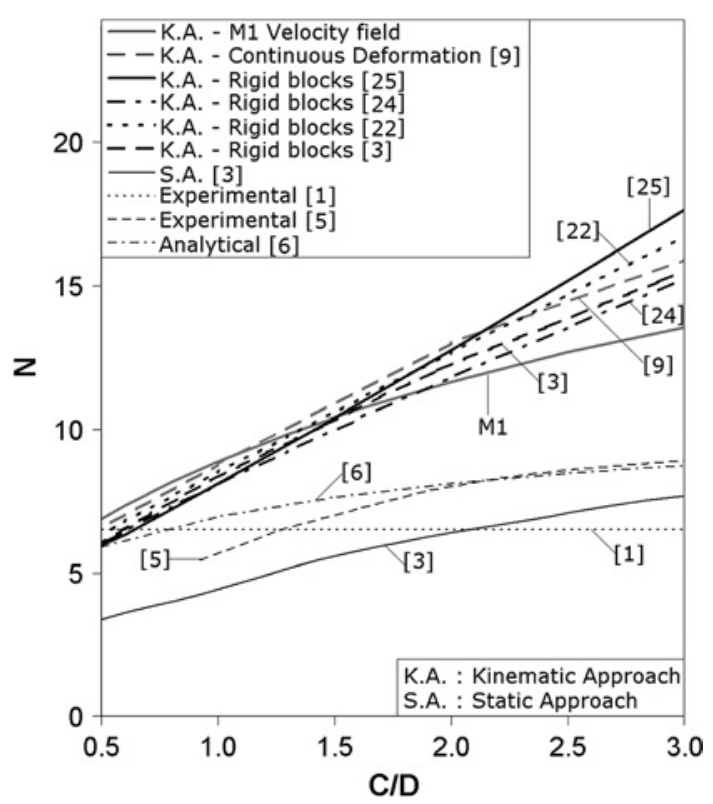

Figure 14. Critical load factor $N$ as provided by the M1 velocity field and by other authors. 
For the M2 velocity field, the classical load factor defined in Equation (29) is not able to assess the face stability because the coefficient $\mathrm{N}_{\gamma}$ related to the soil weight was not found to remain equal to H/D. This new phenomenon arises from the fact that a non-symmetrical velocity field was used in the analysis. For all the rigid block failure mechanisms of the literature $[3,16,22,24,25]$ and for the M1 velocity field presented in this paper, the velocity field at the tunnel face was symmetrical, which means that one half of the flux of velocity crosses the tunnel face above its centre, and the other half crosses the tunnel face below its centre. This condition allows Equation (31) to be valid and makes the computation of the collapse and blowout pressures much simpler. For the M2 velocity field, the point of maximum velocity at the tunnel face is moved at a distance $0.4 \mathrm{D}$ from the face centre (downwards and upwards for the collapse and blowout, respectively), and the velocity field is not symmetrical any more. In this case, the limit pressures are not only defined by $N=N_{\mathrm{c}}$, but by both $N_{\mathrm{c}}$ and $N_{\gamma}$. Moreover, because the position of the point of maximum velocity is different for collapse and blowout, the parameters $N_{\mathrm{c}}$ and $N_{\gamma}$ are different for these two cases. For the two above reasons, the M2 velocity field cannot be compared with the failure mechanisms that make use of only the load factor $N$. Figures 15 and 16 therefore present the comparison in terms of the critical pressures $\sigma_{\mathrm{c}}$ and $\sigma_{\mathrm{b}}$, respectively, for a tunnel of diameter $\mathrm{D}=10 \mathrm{~m}$ in a purely cohesive soil with a unit weight $\gamma=18 \mathrm{kN} / \mathrm{m}^{3}$ and for two values of $c_{\mathrm{u}}(20$ and $30 \mathrm{kPa})$. The numerical results obtained with the FLAC ${ }^{3 \mathrm{D}}$ model are plotted as well. It appears that the M2 velocity field (which significantly improves the best existing upper-bound solutions for collapse pressures) is the one, which compares the best with the numerical model. The values of $\sigma_{\mathrm{c}}$ provided by M2 are very satisfying for $\mathrm{C} / \mathrm{D}<1.5$ and are a bit less good for higher values of $C / D$. For the blowout case, the M1 and M2 velocity fields provide similar values of $\sigma_{\mathrm{b}}$, and these values compare reasonably well with the numerical results.

Using the results provided by the M2 velocity field, a design chart is provided in Figure 17 for practical use in geotechnical engineering. This chart gives the values of the dimensionless coefficients $N_{\gamma}$ and $N_{\mathrm{c}}$ versus $C / D$ for both cases of collapse and blowout. The critical pressures of collapse and blowout can then be easily deduced using Equation (18). To minimize the error that may result from the reading of the values of the coefficients $N_{\gamma}$ and $N_{\mathrm{c}}$ from Figure 17, the values of these coefficients are provided in Table I to be easily used by practitioners. Notice finally that the application of Equation (18) leads to values of the collapse and blowout pressures that are not affected by the error induced by the classical superposition method because the method does not involve any optimization of the velocity field with respect to its geometrical parameters.
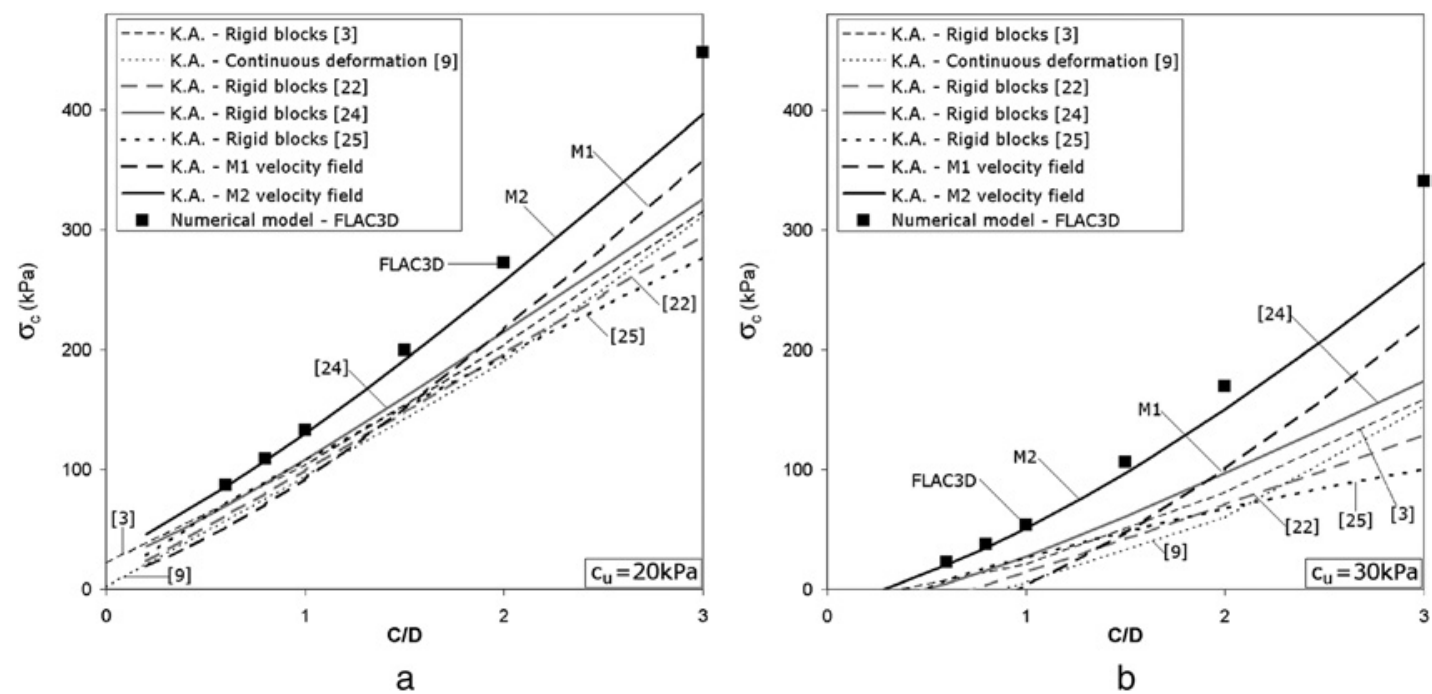

Figure 15. Critical collapse pressures as provided by the existing kinematical approaches, by the present velocity fields $\mathrm{M} 1$ and $\mathrm{M} 2$, and by numerical simulations using FLAC $^{3 \mathrm{D}}$. 


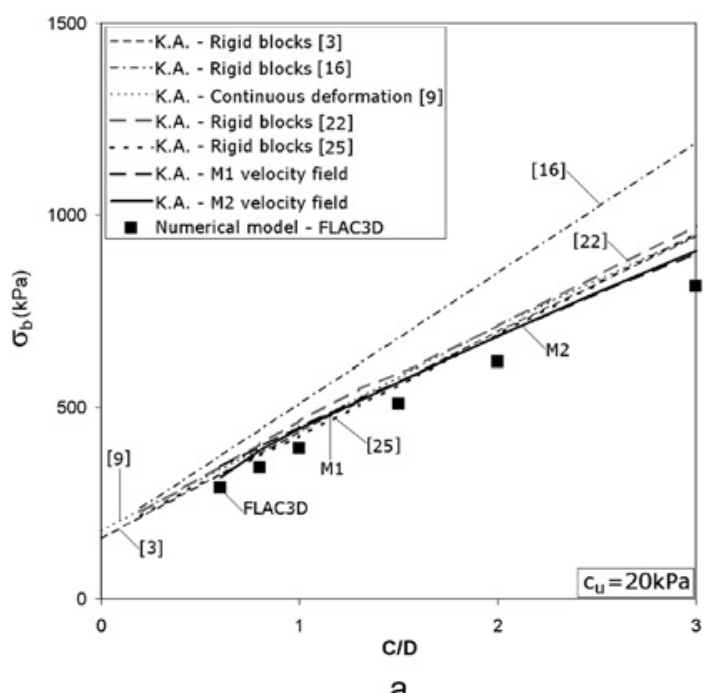

a

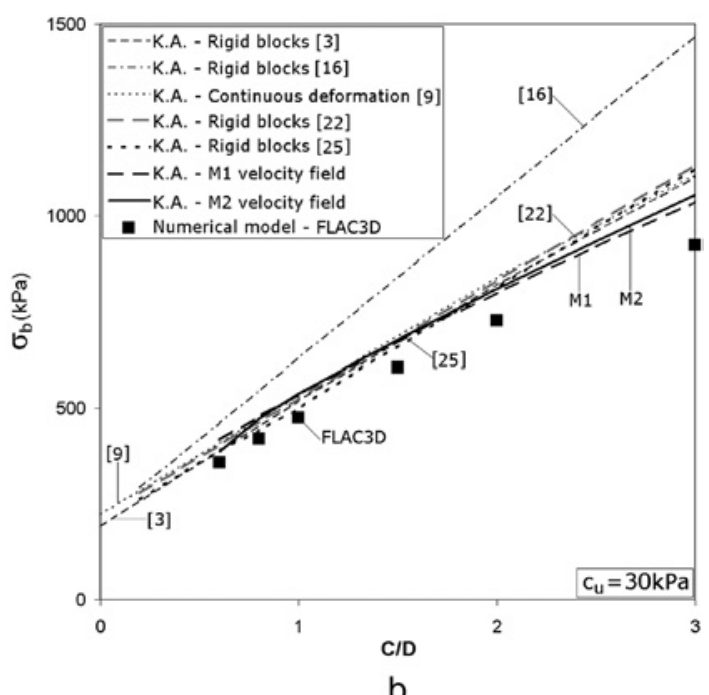

b

Figure 16. Critical blowout pressures as provided by the existing kinematical approaches, by the present velocity fields M1 and M2, and by numerical simulations using FLAC ${ }^{3 \mathrm{D}}$.

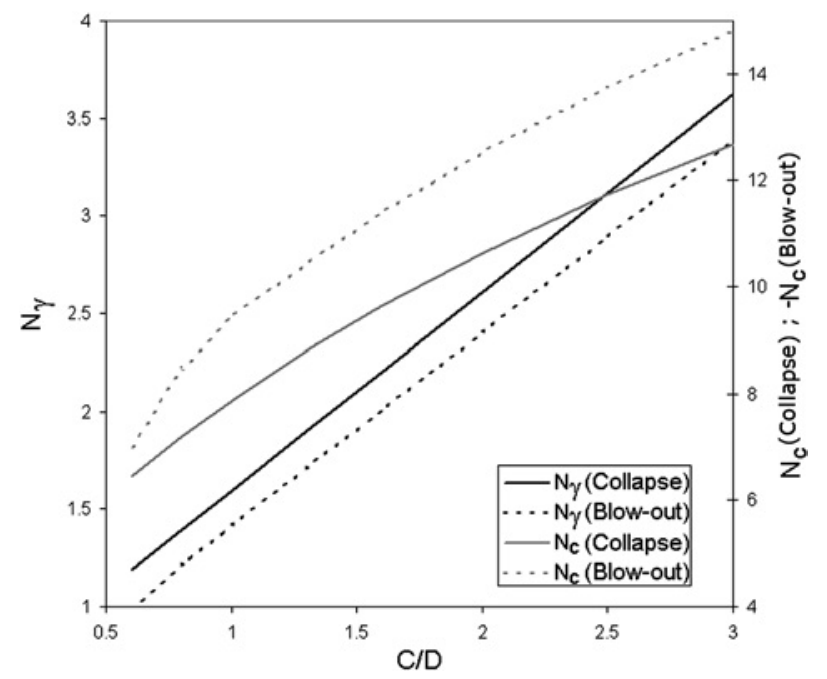

Figure 17. Design chart for the computation of the critical collapse and blowout pressures of a tunnel face in purely cohesive soil.

Table I. Dimensionless parameters $N_{\gamma}$ and $N_{\mathrm{c}}$ for practical design of face pressure.

\begin{tabular}{ccccc}
\hline$C / D$ & $N_{\gamma}$ (Collapse) & $N_{\mathrm{c}}$ (Collapse) & $N_{\gamma}$ (Blowout) & $N_{\mathrm{c}}$ (Blowout) \\
\hline 0.6 & 1.19 & 6.45 & 0.98 & -7.02 \\
0.8 & 1.39 & 7.19 & 1.22 & -8.47 \\
1.0 & 1.59 & 7.87 & 1.42 & -9.43 \\
1.3 & 8.81 & 1.71 & -10.44 \\
1.6 & 1.90 & 9.64 & 2.01 & -11.40 \\
2.0 & 2.20 & 10.64 & 2.40 & -12.53 \\
2.5 & 2.61 & 11.73 & 2.90 & -13.75 \\
3.0 & 3.12 & 12.68 & 3.39 & -14.80 \\
\hline
\end{tabular}




\section{CONCLUSION}

To assess the range of the acceptable face pressure, which has to be applied to a tunnel face in a purely cohesive soil to ensure its stability, two new velocity fields were developed in the framework of the kinematical theorem of limit analysis. Both velocity fields are able to deal with the soil collapse and blowout. They are kinematically admissible and do not include velocity discontinuities. This choice was made after the observation of the failures that were obtained numerically and experimentally in purely cohesive soils. In fact, these failures were not consistent with the existing analytical failure mechanisms based on the motion of rigid blocks with velocity discontinuity surfaces. The first velocity field used in this paper assumes a toric envelope and a symmetrical parabolic profile of the axial velocity at the tunnel face whose maximum value is located at the tunnel centre. In a second step, this velocity field was improved by moving the point of maximal velocity downwards and upwards for the soil collapse and blowout, respectively. The computation of all the components of these velocity fields was performed numerically by implementing a finite difference scheme in a toric mesh because of the complexity of the mathematical expressions involved in these computations. The numerical results have shown that this second velocity field provides critical collapse and blowout pressures that compare reasonably well with the ones obtained by means of numerical simulations using FLAC $^{3 \mathrm{D}}$ but for a much smaller computational cost. Furthermore, this velocity field significantly improves the best existing upper-bound solutions of the collapse pressure. A design chart based on this velocity field is provided for a quick estimate of the critical collapse and blowout pressures in practice.

\section{APPENDIX A: VELOCITY FLUX THROUGH A PLANE $\Pi_{\beta}$ OF THE M1 VELOCITY FIELD}

This appendix aims at demonstrating that the velocity flux of the M1 velocity field through a plane $\Pi_{\beta}$, which may be obtained by integration of the velocity profile over this plane, is constant and independent from the angle $\beta$ of this plane. The velocity vector has three components in the toric system of axis. The component $v_{\beta}$ is normal to the plane $\Pi_{\beta}$, and the two other components $v_{\mathrm{r}}$ and $v_{\theta}$ belong to this plane. The integration of the velocity through $\Pi_{\beta}$ is therefore equal to the one of the $v_{\beta}$ component. This component is expressed by

$$
\left.v_{\beta}(\beta, r, \theta)=v_{m}(\beta) \cdot f(r, \beta)=v_{m}(\beta) \cdot 1-\frac{r^{2}}{[R(\beta)]^{2}}\right)
$$

with:

$$
v_{m}(\beta)=\left(\frac{R_{i}^{2}}{[R(\beta)]^{2}}\right)
$$

The flux of the velocity through the plane $\Pi_{\beta}$ is therefore equal to

$$
F_{\beta}=\iint_{\Pi \beta} v_{\beta}(\beta, r, \theta) d S
$$

In this expression, $d S$ is a small surface element belonging to $\Pi_{\beta}$. Because the velocity components are null outside the envelope of the velocity field, one may write

$$
F_{\beta}=\int_{0}^{R(\beta)} \int_{0}^{2 \pi} v_{\beta}(\beta, r, \theta) \cdot r \cdot d \theta \cdot d r
$$




$$
\begin{gathered}
\left.F_{\beta}=\int_{0}^{R(\beta)} \int_{0}^{2 \pi}\left(\frac{R_{i}^{2}}{[R(\beta)]^{2}}\right) \cdot 1-\frac{r^{2}}{[R(\beta)]^{2}}\right) \cdot r \cdot d \theta \cdot d r \\
\left.\left.F_{\beta}=2 \pi \cdot \frac{R_{i}^{2}}{[R(\beta)]^{2}}\right) \int_{0}^{R(\beta)} 1-\frac{r^{2}}{[R(\beta)]^{2}}\right) \cdot r \cdot d r \\
\left.F_{\beta}=2 \pi \cdot\left(\frac{R_{i}^{2}}{[R(\beta)]^{2}}\right) \cdot \frac{[R(\beta)]^{2}}{2}-\frac{[R(\beta)]^{4}}{4[R(\beta)]^{2}}\right)
\end{gathered}
$$

And finally:

$$
F_{\beta}=R_{i}^{2} \cdot \frac{\pi}{2}
$$

The flux of velocity of the $\mathrm{M} 1$ velocity field through the plane $\Pi_{\beta}$ is therefore constant and independent from the angle $\beta$.

\section{APPENDIX B: GEOMETRY OF A MESH ELEMENT}

The surfaces of the six facets of an elementary zone of the mesh (Figure 8) are given by

$$
\left\{\begin{array}{l}
S_{1} \approx\left(r-\delta_{r} / 2\right) \cdot \delta_{\theta} \times\left(R_{f}-\left(r-\delta_{r} / 2\right) \cdot \cos \theta\right) \cdot \delta_{\beta} \\
S_{1}^{\prime} \approx\left(r+\delta_{r} / 2\right) \cdot \delta_{\theta} \times\left(R_{f}-\left(r+\delta_{r} / 2\right) \cdot \cos \theta\right) \cdot \delta_{\beta} \\
S_{2}=S_{2}^{\prime} \approx \delta_{r} \times\left(R_{f}-r \cdot \cos \theta\right) \cdot \delta_{\beta} \\
S_{3}=S_{3}^{\prime} \approx \delta_{r} \times r \cdot \delta_{\theta}
\end{array}\right.
$$

The average dimensions of this element of the mesh are

$$
\begin{aligned}
& e_{r}=\delta_{r} \\
& e_{\theta}=r \cdot \delta_{\theta} \\
& e_{\beta}=\left(R_{f}-r \cdot \cos \theta\right) \cdot \delta_{\beta}
\end{aligned}
$$

Its volume may be approximated by

$$
\delta V=e_{r} \times e_{\theta} \times e_{\beta}
$$

Points $P_{\mathrm{i}}$ and $P_{\mathrm{i}}$ ' are the centres of the facets $S_{\mathrm{i}}$ and $S_{\mathrm{i}}$ ', respectively, and their coordinates are expressed by

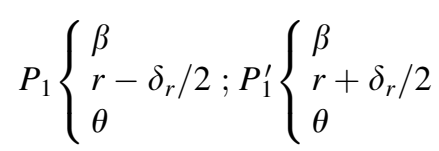




$$
\begin{aligned}
& P_{2}\left\{\begin{array}{l}
\beta \\
r \\
\theta-\delta_{\theta} / 2
\end{array} ; P_{2}^{\prime}\left\{\begin{array}{l}
\beta \\
r \\
\theta+\delta_{\theta} / 2
\end{array}\right.\right. \\
& P_{3}\left\{\begin{array}{l}
\beta-\delta_{\beta} / 2 \\
r \\
\theta
\end{array} ; P_{3}^{\prime}\left\{\begin{array}{l}
\beta+\delta_{\beta} / 2 \\
r \\
\theta
\end{array}\right.\right.
\end{aligned}
$$

\section{APPENDIX C: RATE OF ENERGY DISSIPATION}

The tensor of strain rate is given by

$$
\dot{\varepsilon}=\left[\begin{array}{ccc}
\frac{\partial v_{\beta}}{\partial e_{\beta}} & \frac{1}{2}\left(\frac{\partial v_{\beta}}{\partial e_{r}}+\frac{\partial v_{r}}{\partial e_{\beta}}\right) & \frac{1}{2}\left(\frac{\partial v_{\beta}}{\partial e_{\theta}}+\frac{\partial v_{\theta}}{\partial e_{\beta}}\right) \\
\frac{1}{2}\left(\frac{\partial v_{\beta}}{\partial e_{r}}+\frac{\partial v_{r}}{\partial e_{\beta}}\right) & \frac{\partial v_{r}}{\partial e_{r}} & \frac{1}{2}\left(\frac{\partial v_{r}}{\partial e_{\theta}}+\frac{\partial v_{\theta}}{\partial e_{r}}\right) \\
\frac{1}{2}\left(\frac{\partial v_{\beta}}{\partial e_{\theta}}+\frac{\partial v_{\theta}}{\partial e_{\beta}}\right) & \frac{1}{2}\left(\frac{\partial v_{r}}{\partial e_{\theta}}+\frac{\partial v_{\theta}}{\partial e_{r}}\right) & \frac{\partial v_{\theta}}{\partial e_{\theta}}
\end{array}\right]
$$

By using the assumptions made on the axial and orthoradial components of the velocity vector, Equation (C1) may be re-written as follows:

$$
\dot{\varepsilon}=\left[\begin{array}{ccc}
\frac{\partial v_{\beta}}{\partial e_{\beta}} & \frac{1}{2}\left(\frac{\partial v_{\beta}}{\partial e_{r}}+\frac{\partial v_{r}}{\partial e_{\beta}}\right) & 0 \\
\frac{1}{2}\left(\frac{\partial v_{\beta}}{\partial e_{r}}+\frac{\partial v_{r}}{\partial e_{\beta}}\right) & \frac{\partial v_{r}}{\partial e_{r}} & \frac{1}{2} \frac{\partial v_{r}}{\partial e_{\theta}} \\
0 & \frac{1}{2} \frac{\partial v_{r}}{\partial e_{\theta}} & 0
\end{array}\right]
$$

The terms of this tensor can be easily computed numerically using a finite difference explicit scheme as follows:

$$
\begin{aligned}
\frac{\partial v_{\beta}}{\partial e_{\beta}} & =\frac{v_{\beta}\left(P_{3}\right)-v_{\beta}\left(P_{3}^{\prime}\right)}{e_{\beta}} \\
\frac{\partial v_{\beta}}{\partial e_{r}} & =\frac{v_{\beta}\left(P_{1}^{\prime}\right)-v_{\beta}\left(P_{1}\right)}{e_{r}} \\
\frac{\partial v_{r}}{\partial e_{r}} & =\frac{v_{r}\left(P_{1}^{\prime}\right)-v_{r}\left(P_{1}\right)}{e_{r}} \\
\frac{\partial v_{r}}{\partial e_{\beta}} & =\frac{v_{r}\left(P_{3}\right)-v_{r}\left(P_{3}^{\prime}\right)}{e_{\beta}}
\end{aligned}
$$




$$
\frac{\partial v_{r}}{\partial e_{\theta}}=\frac{v_{r}\left(P_{2}\right)-v_{r}\left(P_{2}^{\prime}\right)}{e_{\theta}}
$$

\section{APPENDIX D: COMPUTATION OF THE PARAMETER $r_{\max }$}

For a given value of the angular parameter $\theta$ in a $\Pi_{\beta}$ plane, the parameter $r_{\max }(\beta, \theta)$ is the distance between the point $E_{\beta}$ (belonging to the circle () and the external surface of the M2 velocity field. This parameter is chosen to be

$$
r_{\max }(\beta, \theta)=r_{\max }(0, \theta) \cdot \frac{R_{\beta}}{R_{i}}
$$

With

$$
\begin{gathered}
R_{i}=D / 2+L_{1} \\
R_{\beta}=R_{i}+\left(R_{f}-R_{i}\right) \frac{\beta}{\pi / 2}
\end{gathered}
$$

Thus, for any value of the angle $\theta$, the distance $r_{\max }$ linearly increases with $\beta$, starting with a value $r_{\max }(0, \theta)$ at the tunnel face. This increase is linked with the one of $R_{\beta}$ which linearly increases from $R_{\mathrm{i}}$ to $R_{\mathrm{f}}$. The value of $r_{\max }$ in the plane $\Pi_{0}$ of the tunnel face is obtained by the following expression:

$$
r_{\max }(0, \theta)=\sqrt{\left(z_{i}+L_{1}\right)^{2}+x_{i}^{2}}
$$

The distances $x_{\mathrm{i}}$ and $z_{\mathrm{i}}$ are represented in Figure 11, and their expressions are

$$
\begin{gathered}
x_{i}=\sqrt{-z_{i}^{2}+D^{2} / 4} \\
z_{i}=\frac{-B \pm \sqrt{\Delta}}{2 A}
\end{gathered}
$$

with the intermediate variables:

$$
\begin{gathered}
\Delta=B^{2}-4 A \cdot C \\
A=1+\tan ^{2}(\pi / 2-\theta) \\
B=2 \cdot L_{1} \\
C=L_{1}^{2}-\frac{D^{2} \cdot \tan ^{2}(\pi / 2-\theta)}{4}
\end{gathered}
$$

\section{REFERENCES}

1. Broms BB, Bennermark H. Stability of clay at vertical openings. Journal of the Soil Mechanics and Foundation Engineering 1967; 193(SM1):71-94.

2. Mair RJ. Centrifugal modelling of tunnel construction in soft clay. PhD thesis, University of Cambridge, 1969.

3. Davis EH, Gunn MJ, Mair RJ, Seneviratne HN. The stability of shallow tunnels and underground openings in cohesive material. Geotechnique 1980; 30(4):397-416. 
4. Schofield AN. Cambridge geotechnical centrifuge operations. Geotechnique 1980; 30(3):227-268.

5. Kimura T, Mair RJ. Centrifugal testing of model tunnels in clay. Proc. 10th Int. Conf. of Soil Mechanics and Foundatio Engineering, Stockholm, Rotterdam: Balkema, 1, 1981, 319-322.

6. Ellstein AR. Heading failure of lined tunnels in soft soils. Tunnels and Tunnelling 1986:51-54.

7. Augarde CE, Lyamin AV, Sloan SW. Stability of an undrained plane strain heading revisited. Computers and Geotechnics 2003; 30:419-430.

8. Osman AS, Mair RJ, Bolton MD. On the kinematics of 2D tunnel collapse in undrained clay. Geotechnique 2006; 56 (9):585-595.

9. Klar A, Osman AS, Bolton M. 2D and 3D upper bound solutions for tunnel excavation using 'elastic' flow fields. International Journal for Numerical and Analytical Methods in Geomechanics 2007; 31(12):1367-1374.

10. Chambon P, Corté JF. Shallow tunnels in cohesionless soil: Stability of tunnel face. Journal of Geotechnical Engineering ASCE 1994; 120(7):1148-1165.

11. Kamata $\mathrm{H}$, Mashimo $\mathrm{H}$. Centrifuge model test of tunnel face reinforcement by bolting. Tunnelling and Underground Space Technology 2003; 18:205-212.

12. Takano, D, Otani, J, Nagatani, H, Mukunoki, T. Application of X-ray CT boundary value problems in geotechnical engineering - Research on tunnel face failure. Geocongress, GSP $N^{\circ} 2006,1-6$.

13. Kirsch A. Experimental investigation of the face stability of shallow tunnels in sand. Acta Geotechnica 2010; 5(1):43-62.

14. Horn N. Horizontaler erddruck auf senkrechte abschlussflächen von tunnelröhren. Landeskonferenz der ungarischen tiefbauindustrie 1961:7-16.

15. Atkinson JH, Potts DM. Stability of a shallow circular tunnel in cohesionless soil. Geotechnique 1977; 27(2):203-215.

16. Leca E, Dormieux L. Upper and lower bound solutions for the face stability of shallow circular tunnels in frictional material. Geotechnique 1990; 40(4):581-606.

17. Eisenstein AR, Ezzeldine O. The role of face pressure for shields with positive ground control. In Tunneling and ground conditions. Balkema: Rotterdam, 1994; 557-571.

18. Anagnostou G, Kovari K. Face stability conditions with earth-pressure-balanced shields. Tunnelling and Underground Space Technology 1996; 11(2):165-173.

19. Broere W. Face stability calculation for a slurry shield in heterogeneous soft soils. Proc. of the world tunnel congress 98 on tunnels and metropolises, Sao Paulo, Rotterdam:Balkema, 1, 1998, 215-218.

20. Vermeer PA, Ruse NM, Marcher T. Tunnel heading stability in drained ground. Felsbau 2002; 20(6):8-18.

21. Kolymbas D. Tunnelling and Tunnel Mechanics. Springer: Berlin, 2005.

22. Mollon G, Dias D, Soubra A-H. Probabilistic analysis and design of circular tunnels against face stability. International Journal of Geomechanics, ASCE 2009a; 9(6):237-249.

23. Mollon G, Dias D, Soubra A-H. Probabilistic analysis of circular tunnels in homogeneous soils using response surface methodology. Journal of Geotechnical and Geoenvironmental Engineering, ASCE 2009b; 135(9):1314-1325.

24. Mollon G, Dias D, Soubra A-H. Face stability analysis of circular tunnels driven by a pressurized shield. Journal of Geotechnical and Geoenvironmental Engineering, ASCE 2010; 136(1):215-229.

25. Mollon G, Dias D, Soubra A-H. Rotational failure mechanisms for the face stability analysis of tunnels driven by pressurized shields. International Journal for Numerical and Analytical Methods in Geomechanics 2011; 35 (12):1363-1388.

26. FLAC ${ }^{3 \mathrm{D}}$. Fast Lagrangian Analysis of Continua. ITASCA Consulting Group, Inc.: Minneapolis, 1993.

27. Chen WF. Limit analysis and soil plasticity. Elsevier: Amsterdam, 1975. 